\title{
Análise de Dados de Língua Eletrônica baseada em redes SOM 1D*
}

\author{
L. P. O. Sousa * D. D. Ferreira * K. L. Fukushima * \\ J. E. Oliveira* \\ * Departamento de Engenharia, Universidade Federal de Lavras, MG \\ (e-mail: lpsousa14@gmail.com; danton@ufla.br; katiafuku@gmail.com; \\ juliano.oliveira@ufla.br).
}

\begin{abstract}
The design of devices that resemble biological recognition systems, inspired by the functioning of human organs, have made important advances in recent decades. As a result, interest in the concept of electronic languages has increased considerably in recent years. This study uses this type of device in order to identify different samples of flavor enhancers, such as Glutamate, Guanylate and Inosinate. The applied methodology involves self-organizing maps, an unusual tool in this area. The best results reached 95,4\% accuracy.

Resumo: O projeto de dispositivos que se assemelham a sistemas de reconhecimento biológico, inspirados no funcionamento de órgãos humanos, tiveram avanços importantes nas últimas décadas. Em consequência disso, o interesse no conceito de línguas eletrônicas aumentou consideravelmente nos últimos anos. Este estudo utiliza esse tipo de dispositivo com o intuito de identificar diferentes amostras de realçadores de sabor, como Glutamato, Guanilato e Inosinato. A metodologia aplicada envolve redes do tipo mapas auto-organizáveis, ferramenta pouco usual nessa área. Os melhores resultados atingiram 95,4\% de precisão.
\end{abstract}

Keywords: Self Organizing Maps; E-tongue; Clustering. Palavras-chaves: Mapas Auto Organizáveis; Língua Eletrônica; Classificação.

\section{INTRODUÇÃO}

Os primeiros sistemas inspirados nos órgãos olfativos e gustativos foram propostos na década de 1980. A língua eletrônica (LE) é um sistema que usa técnicas sensoriais e instrumentais para determinar sabores ou substâncias presentes em amostras aquosas. Em consequência disso, o interesse nesse conceito aumentou consideravelmente nos últimos anos (Nery and Kubota (2016); Wei and Wang (2014)).

Basicamente, as LEs são compostas por unidades de coleta de informações (arranjos sensoriais), e no processamento dessas informações, utilizam-se técnicas matemáticas. Essas informações coletadas podem ser definidas como a "impressão digital" da amostra e correspondem ao conjunto de informações valiosas como reconhecimento, identificação ou quantificação de constituintes da amostra (Nery and Kubota (2016); Wei and Wang (2014)).

Ao passar dos anos houve avanços importantes no projeto desses sensores, normalmente direcionados para a implementação de novos conceitos, projetos ou configurações. Apesar de ser uma aplicação recente da área sensorial, já existe uma grande quantidade de aplicações envolvendo LE em diversos segmentos, como na indústria química, medicinal e alimentar (Cetó et al. (2015); Nery and Kubota (2016); Wei et al. (2013)).

\footnotetext{
* Agradecimento à CNPq (Conselho Nacional de Desenvolvimento Científico e Tecnológico) e a FAPEMIG (Fundação de Amparo á Pesquisa do Estado de Minas Gerais).
}

Por meio de uma pesquisa rápida na literatura, nota-se que métodos como Principal Component Analysis (PCA) e Partial Least Squares (PLS) são utilizados com bastante frequência em análises de dados de LE, porém, essas técnicas possuem algumas limitações.

O PCA não é a ferramenta mais adequada para ser usada na classificação de objetos, pois realiza uma transformação linear e ortogonal nos dados, em que no novo espaço gerado pelo PCA os dados são descorrelacionados. Em algumas situações essa descorrelação não é suficiente para agrupar as classes adequadamente. Ao usar PLS, as classes são divididas usando partições lineares no espaço de classificação que podem ser problemáticas se a separação entre classes for não linear (Zielinski et al. (2014)). Portanto, métodos mais sofisticados e não lineares são requeridos.

O objetivo principal dessa pesquisa é elaborar modelos de classificação que sejam capazes de discriminar amostras de diferentes realçadores de sabor. Os modelos de classificador elaborados utilizam redes de Kohonen, também conhecidas como Mapas Auto-Organizáveis (Self-Organization Maps - SOM). Outro objetivo é identificar qual arquitetura de eletrodo usado na LE obtém a maior descriminância dessas substâncias.

O restante do artigo está organizado da seguinte forma. A Seção 2 traz a revisão da literatura com o intuito de exemplificar os estudos que têm sido realizados nessa área de pesquisa. A Seção 3 é destinada à apresentação do experimento para obtenção dos dados e os detalhes e definições dos métodos usados. Os resultados e as análises 
alcançados são expostas na Seção 4. E por fim, na Seção 5, as conclusões do trabalho e os possíveis trabalhos futuros são destacados.

\section{ESTADO DA ARTE}

Devido à grande quantidade de aplicações possíveis relacionadas a esse tema, podem ser encontrados diversos estudos realizados na literatura. Normalmente, na análise de LE, os classificadores são comumente usados para reconhecer ou classificar amostras, como em Lu et al. (2016) que apresenta uma abordagem para caracterização e quantificação de atributos do sabor do arroz, como suavidade, adesividade, doçura e aroma. Empregando uma LE voltamétrica e combinando PCA e Fast Fourier Transform (FFT) em seus modelos.

O trabalho de Gutiérrez-Capitán et al. (2014) apresenta uma LE para análise de vinhos, com o uso de ferramentas de processamento de dados, como PCA e PLS. Os vinhos foram classificados de acordo com suas características, como: variedade de uva, origem geográfica e ano.

Outra abordagem foi apresentada em Cetó et al. (2015) para discriminar amostras de vinhos de diferentes origens e os dados foram pré-processados com FFT e classificadas com Linear Discriminant Analysis (LDA) e rede neural do tipo Mult Layer Perceptron (MLP). Kang et al. (2014) projetaram um sistema de LE para diferenciar vinhos de arroz coreano fabricados de nove cultivares de arroz, usando o método PLS.

A pesquisa de Liu et al. (2013) introduz Random Forest (RF) no processamento de dados de LE potenciométrica, projetados para reconhecimento de tipo e marca de bebida de laranja e vinagre chinês. PCA foi utilizada para visualizar a distribuição e a classificação foi feita com métodos MLP e Support Vector Machine (SVM).

Em Saha et al. (2016) uma LE é usada na caracterização de amostras de chá de diferentes qualidades, fazendo uso de SVM e MLP. O trabalho de Sharma and Ugale (2015) descreve uma maneira eficiente de identificar líquidos, como cerveja, chá, café, mel, leite e outros. por meio de um sistema de LE. Para isso, é utilizado o algoritmo Linear Vector Quantization (LVQ) e MLP.

O artigo de Ghosh et al. (2016) mostra uma LE implementada para detectar duas toxinas: Aflatoxina B1 (AFB1) e Ocratoxina A (OTA). Os parâmetros extraídos foram processados usando PCA e PLS. Outra aplicação envolvendo caracterização química é apresentada em Silva and Paixão (2017), onde uma LE voltamétrica foi usada sobre agentes de corte adicionados à cocaína (como, procaína, benzocaína, fenacetina, levamisol e cafeína) para fins de discriminação e classificação. As informações foram analisadas com PCA e K-Nearest Neighbor (KNN).

Há poucos relatos do uso de rede SOM no campo de LE. $\mathrm{Na}$ revisão feita, um sistema de LE foi desenvolvida e usada para a análise qualitativa de cinco diferentes tipos de águas minerais. Os dados originais adquiridos foram otimizados pela técnica Independent Component Analysis (ICA) e LDA, e então três modelos foram projetados para classificar as amostras, usando LVQ, SOM e um combinando ICA com LVQ (Men et al. (2009)). Outro estudo que usa rede SOM e MLP foi apresentado em Breijo et al. (2013) relata a utilização de uma LE voltamétrica para a detecção e discriminação de diferentes concentrações de 2 , 4 e 6 trinitrotolueno (TNT) em misturas de acetonitrilo.

\section{MATERIAIS E MÉTODOS}

\subsection{Lingua Eletrônica}

O conceito de LE é baseado nos mecanismos do sistema gustativo do ser humano, a Figura 1 mostra um diagrama simplificado da relação dos mesmos. A língua humana é o órgão responsável por fornecer informações sobre o gosto, textura e temperatura dos alimentos. Através do conceito de seletividade global, a língua é capaz de identificar os sabores básicos. Esta percepção é possível devido os receptores gustativos presentes na língua, que por meio de estímulos, os receptores geram sinais elétricos que são transmitidos ao cérebro e assim, interpretados pelo mesmo (Deisingh and abnd Michael Thompson (2004)).

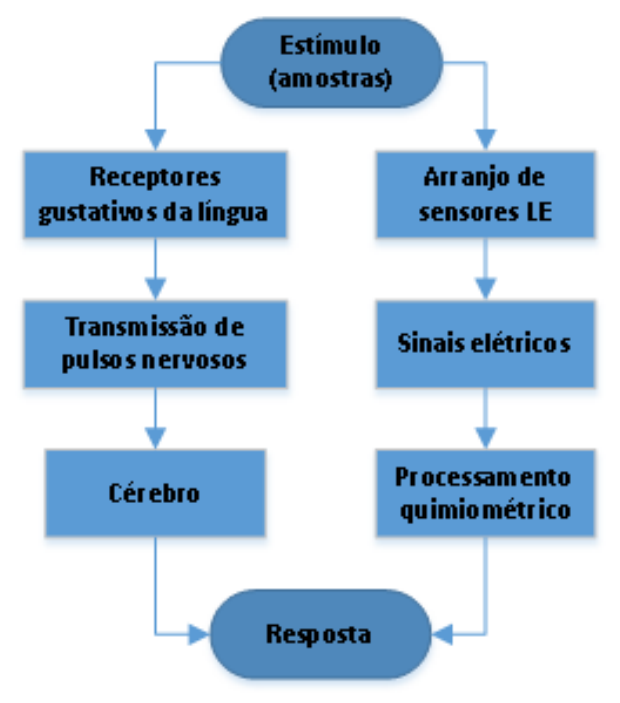

Figura 1. Comparação entre o sistema biológico e o sistema eletrônico.

Baseado nisso, a LE instrumento analítico baseado em uma série de sensores químicos de baixa seletividade e com o auxílio de processamentos matemáticos são capazes de caracterizar amostras líquidas complexas (Wei et al. (2013); Peris and Escuder-Gilabert (2013)).

As LE desenvolvidas nos últimos anos podem ser classificadas em três tipos: as que são baseados em eletrodos de espectroscopia potenciométrica, voltamétrica e de impedância (Wei and Wang (2014); Liu et al. (2013)). De modo geral, uma LE é composta por três elementos: (1) uma matriz de sensores químicos com diversos seletivos, (2) um amplificador e gerador de sinal e (3) um software com o algoritmo apropriado para processar os sinais e analisar os resultados. A Figura 2 ilustra um esquemático de um sistema de LE completo.

A principal vantagem do sistema de LE em comparação aos métodos tradicionais é que eles permitem a implementação de sistemas de medição rápida e de baixo custo, evitando a necessidade de pessoal especializado para realizar as análises (Breijo et al. (2013); Saha et al. (2014)). 


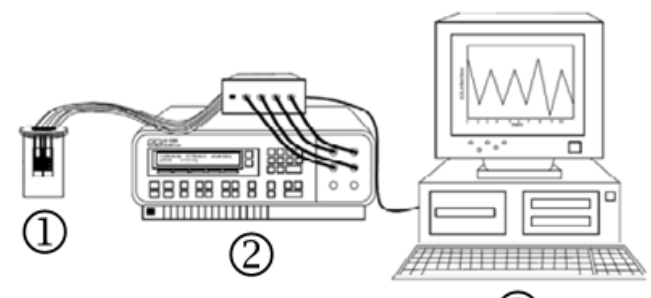

(3)

Figura 2. Elementos de uma LE.

A LE utilizada nesse estudo, foi desenvolvida em Fukushima (2017), onde a mesma tinha como objetivo desenvolver, caracterizar e aplicar sensores nanoestruturados para análise de realçadores de sabor em soluções aquosas. Em sua composição foram utilizados seis eletrodos interdigitados de ouro fabricados sobre lâminas de vidro modificados através da técnica Layer-by-Layer.

Esses eletrodos, foram elaborados de acordo com as arquiteturas apresentadas na Tabela 1, onde PAH é poli(alilamina hidroclorada), rGO é óxido de grafeno reduzido, PANI é poli(anilina), CuTsPc é ftalocianin tetrassulfonada de cobre e AgNPs são nanopartículas de prata.

Tabela 1. Arquiteturas dos eletrodos da LE.

\begin{tabular}{c|c|c}
\hline Eletrodo & Camadas & Arquitetura \\
\hline \hline E1 & 2 tetra camadas & PAH/rGO/PANI/CuTsPc \\
E2 & 2 tetra camadas & PAH/rGO/PAH/CuTsPc \\
E3 & 2 tetra camadas & PANI/AgNPs/PANI/CuTsPc \\
E4 & 2 tetra camadas & PAH/AgNPs/PAH/CuTsPc \\
E5 & 4 bi camadas & PANI/CuTsPc \\
E6 & 4 bi camadas & PAH/CuTsPc \\
\hline
\end{tabular}

Os detalhes sobre o preparo e a caracterização das soluções e dos filmes utilizados podem ser encontrados no estudo realizado em Fukushima (2017).

\subsection{Amostras}

As amostras utilizadas no experimento são compostas por Glutamato Monossódico (GM), Inosinato Dissódico (ID), e uma mistura 1:1 $(50 \% \mathrm{~m} / \mathrm{m})$ de Guanilato Dissódico (GD) com ID, os quais são realçadores de sabor muito utilizados em indústrias alimentícias. Os realçadores de sabor são aditivos alimentares usados para causar um efeito de gosto e sabor mais marcante nos alimentos ao paladar do consumidor Milani (2013).

O GM é um aminoácido não essencial encontrado naturalmente em alimentos e em organismos vivos, como peixes, carnes vermelhas, leite e diversos vegetais. Enquanto o ID e o GD são nucleotídios obtidos a partir da fermentação do amido e podem ser encontrados em sardinhas secas, atum, carnes suína, bovina e de frango ou em alguns cogumelos. Tais aditivos são comumente adicionados aos produtos cárneos, sopas, snacks, temperos e molhos. Esses realçadores proporcionam o quinto sabor básico, o "Umami", que em japonês significa saboroso (Milani (2013)).

Os dados obtidos pela LE foram capturados usando seis unidades sensoriais conectadas nos canais de leituras, as quais foram feitas utilizando os softwares ZPlot 2.6 e ZView 2.6. Os dados foram coletados a partir das medidas de resistência em função da frequência de $10 \mathrm{~Hz}$ a $1 \mathrm{MHz}$.
A estrutura dos dados gerados pela LE utilizada, consiste em cinco variáveis medidas, agrupadas em um vetor linha $x_{k}=\left[x_{k 1}, x_{k 2}, \ldots, x_{k 5}\right]^{T}, x_{k} \in R^{n}$. As variáveis obtidas pela LE foram: Frequência em Hertz (F), Impedância real (Zr), Impedância Imaginária (Zi), Capacitância (C) e Resistência (R).

\subsection{Mapas Auto-Organizáveis}

O modelo de rede de mapas auto-organizáveis (SOM) é um método de rede neural competitiva que aprende a classificar os eventos de entrada conforme os mesmos são agrupados no espaço de entrada. As localizações espaciais (ou coordenadas) dos eventos no espaço de saída são indicativos de características estatísticas inerentes contidos no espaço de entrada (Beale et al. (2012); Asan and Ercan (2012)).

O principal objetivo da rede SOM é transformar um espaço complexo de alta dimensionalidade em um espaço de baixa dimensionalidade, mas tentando preservar suas relações. A rede aprende a distribuição e a topologia dos eventos de entrada em que são usados no treinamento. A formação dessa rede envolve três processos característicos (Beale et al. (2012); Asan and Ercan (2012)).

O primeiro é a concorrência, onde os neurônios de saída competem uns com os outros para melhor representar o evento de entrada particular. O neurônio específico com seus pesos mais semelhantes ao evento de entrada é declarado vencedor da competição. O segundo é a cooperação, em que o neurônio vencedor determina a localização espacial de uma vizinhança de neurônios cooperantes. Assim, compartilhando recursos comuns e estimulando uns aos outros para aprender algo a partir da mesma entrada (Beale et al. (2012); Asan and Ercan (2012)).

E por fim, a adaptação, que em consequência do processo de aprendizagem, os vetores de peso do vencedor e suas unidades vizinhas no mapa são ajustados e os neurônios relevantes tornam-se mais semelhantes ao evento de entrada. Dessa forma, os neurônios que têm uma resposta forte a uma determinada peça de dados de entrada terão uma chance maior de responder aos dados de entrada semelhantes no futuro.

Os pesos são links que conectam os neurônios de entrada com os da saída e são atualizados através do processo de aprendizagem. Em cada etapa de treinamento, um vetor do evento $\mathbf{x}$ do conjunto de dados de entrada é escolhido aleatoriamente e as distâncias entre ele e todos os vetores de peso do SOM são calculadas usando alguma medida de distância. O neurônio vencedor (BMU - Best-Matching Unit), denotado aqui por $c$, é o que satisfaz:

$$
\left\|\mathbf{x}-\mathbf{m}_{c}\right\|=\min _{i}\left\{\left\|\mathbf{x}-\mathbf{m}_{i}\right\|\right\},
$$

onde $\|\cdot\|$ é a norma de distância e $\mathbf{m}$ é o vetor de pesos. Neste estudo foi adotada a norma Euclidiana.

Depois de encontrar o BMU, os vetores de peso são atualizados para se tornarem mais representativos em relação às características do espaço de entrada. Uma determinada vizinhança do BMU também são atualizados para preser- 
var a estrutura tipológica do mapa. Essa atualização da unidade $i$ é:

$$
\mathbf{m}_{i}(t+1)=\mathbf{m}_{i}(t)+\alpha(t) h_{c i}(t)\left[\mathbf{x}(t)-\mathbf{m}_{i}(t)\right],
$$

onde $t$ denota a época. O $\mathbf{x}(t)$ é um vetor de entrada desenhado aleatoriamente a partir do conjunto de dados de entrada na época $t, h_{c i}(t)$ é o núcleo de vizinhança ao redor do BMU $c$ e $\alpha(t)$ é a taxa de aprendizado na época $t$ (Asan and Ercan (2012); Vesanto et al. (2000)).

Todos os processos descritos são repetidos para os dados de treinamento restantes até os pesos convergirem e não haja alterações perceptíveis na camada de saída de baixa dimensão. Os neurônios na camada de saída da rede são dispostos de acordo com uma função topológica (Beale et al. (2012); Asan and Ercan (2012)).

\subsection{Metodologia}

Primeiramente, a aquisição dos dados foi realizada por meio do sistema de língua eletrônica descrito. Em posse desses dados, o próximo passo é o pré-processamento do conjunto de dados obtidos. A técnica empregada foi a normalização dos dados via transformada z-scores, processo que traz benefícios ao banco de dados, como evitar a polarização dos dados. Assim, os dados foram normalizados conforme a equação (Duda et al. (2001)):

$$
\mathbf{x}=\frac{\mathbf{x}_{\text {old }}-\bar{x}}{\sigma}
$$

onde $\mathbf{x}_{\text {old }}$ é o vetor de observações original, $\mathbf{x}$ é o vetor normalizado e $\bar{x}$ e $\sigma$ são a média e o desvio padrão do vetor $\mathbf{x}_{\text {old }}$, respectivamente.

O trabalho foi desenvolvido através da linguagem de programação do MatLab, que é uma linguagem que fornece um poderoso ambiente computacional para aplicações científicas. O uso dessa ferramenta facilita a codificação de algoritmos complexos em comparação com outras linguagens.

A Figura 3 apresenta um fluxograma dos procedimentos que o modelo projetado segui. Observe que os dados préprocessados são separados em dados de treino, teste e validação. Com isso, as redes SOM são criadas com os dados de treino e testadas com os dados de teste. Esse processo é feito diversas vezes com parâmetros diferentes, com o intuito de achar os que proporcionem melhores resultados. No final, o banco de dados de validação é apresentado à rede para verificar a eficiência final e a capacidade de generalização.

Com as redes treinadas, são determinados os BMUs, que são os neurônios vencedores de cada rede. Assim, é feito o cálculo da distância entre os dados de validação, dados que não foram apresentados a rede, e os BMUs. Cada substância é representada pela mesma quantidade de BMUs e a pertinência das novas observações são classificadas conforme essas distâncias calculadas. A observação é atribuída ao BMU que tenha a menor distância. Assim, basta identificar a qual substância o BMU representa. Vale destacar que o modelo conta com uma rede SOM $1 \mathrm{D}$ para cada substância, ou seja, o modelo tem três redes.

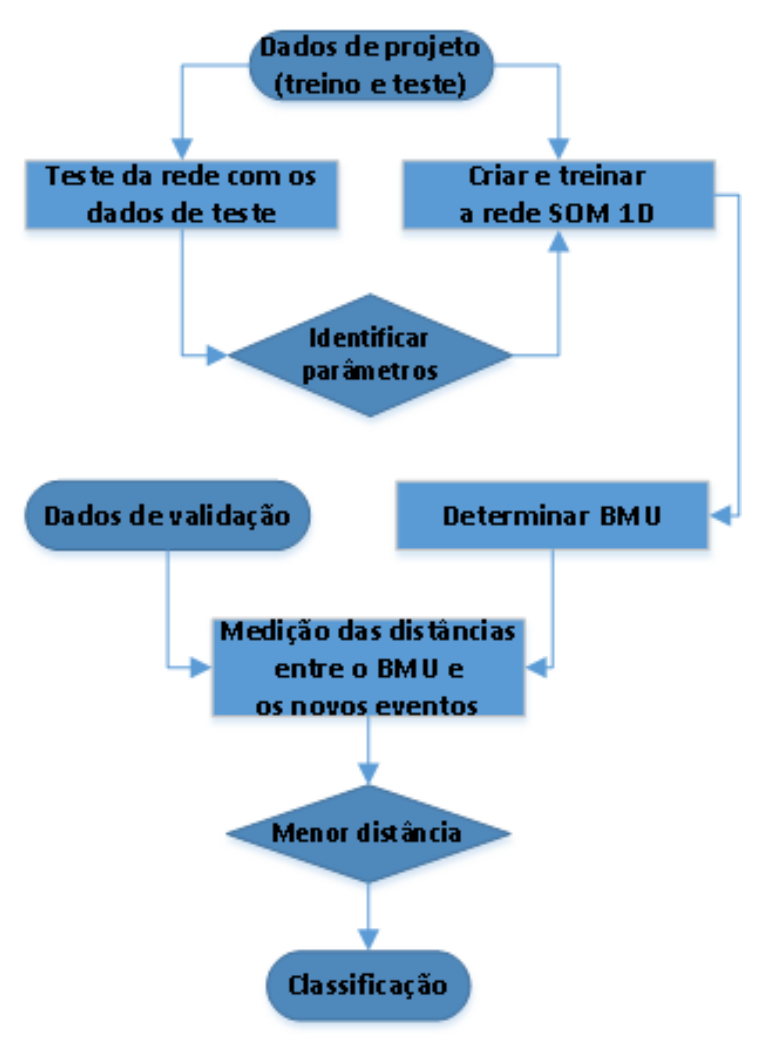

Figura 3. Fluxograma do classificador projetado.

\section{RESULTADOS E DISCUSSÕES}

A Figura 4 mostra o espaço de características formado pela combinação das variáveis $\mathrm{Zr}$ e C, para todas substâncias e de cada arquitetura de eletrodo. Observe que os dados apresentam uma disposição no espaço de características de forma alongada e não linear. Essa disposição característica dos dados é uma das motivações para o uso da técnica de rede SOM 1D.

As redes SOM aprendem a agrupar dados com base na semelhança e topologia. O resultado da interface da rede SOM gerada pelo MatLab é apresentada na Figura 5. Os parâmetros que integram essas redes são (os parâmetros padrões utilizados foram escolhidos conforme as recomendações de Vesanto et al. (2000)):

- As dimensões do mapa, variando em [1 3], [1 10], [1 25], [1 50] e [1 100].

- O número de épocas de treinamento para cobertura inicial do espaço de entrada, definido em 200 épocas.

- O tamanho inicial da vizinhança, usando o valor padrão 3 .

- Como está sendo usado rede SOM 1D, a função de topologia adotada foi o padrão.

- A função de distância do neurônio, tendo as opções dist, linkdist, mandist e boxdist. O melhor desempenho foi usando linkdist.

Um dos métodos que valida a resposta obtida pelo classificador é a validação cruzada, sendo frequentemente usada para estimar a capacidade de generalização de um classificador. Nesse sentido, o método de validação cruzada escolhido foi o hold-out. O método hold-out reserva um conjunto para treinamento de forma aleatória e seu com- 
E1

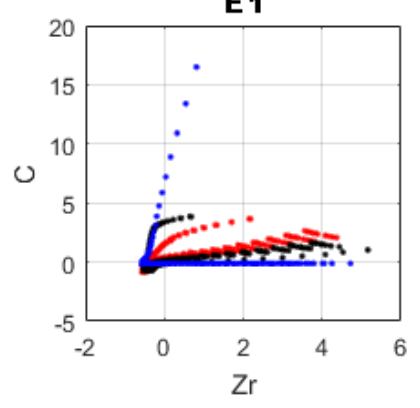

E3

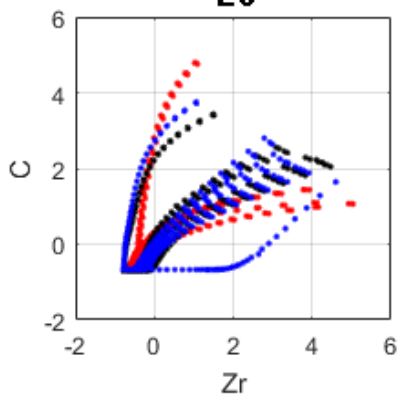

E5

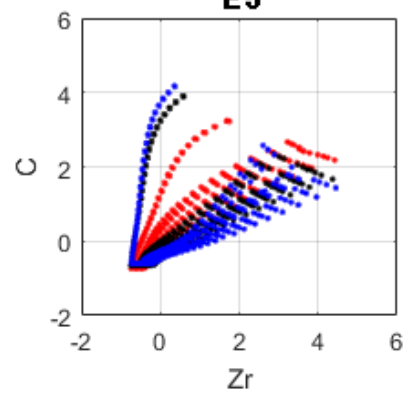

E2

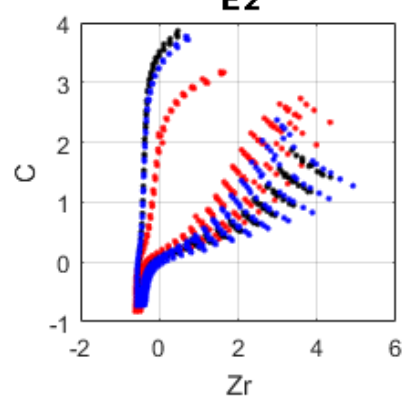

E4

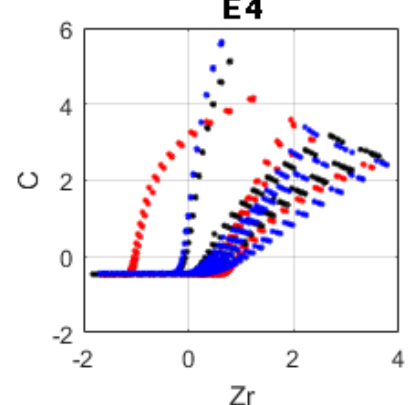

E6

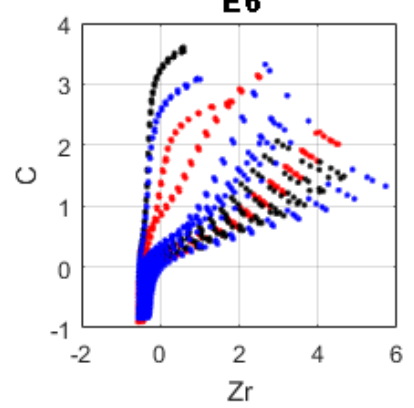

Figura 4. Sinais captados em duas dimensões de cada eletrodo.

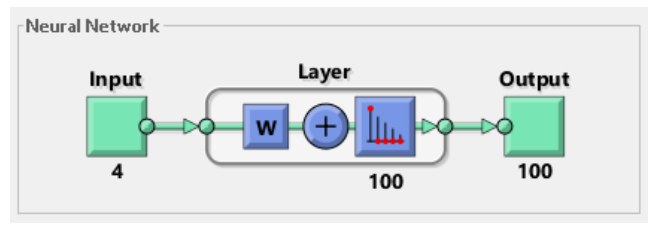

Figura 5. Interface da rede SOM 1D ([1,100]).

plemento para teste. Devido a quantidade de observações, $75 \%$ foi determinado para treino e o restante para teste (Cawley and Talbot (2003)).

Os percentuais podem ser mais confiáveis se o processo de hold-out for repetido com diferentes observações, por isso o conjunto é aleatoriamente selecionada para treinamento, assim garantindo que os conjuntos de treinamento sejam diferentes. Ficou definido que esse processo será aplicado vinte vezes, para gerar uma boa estimação dos resultados. Logo, os percentuais de precisão equivalem a média desses diferentes conjuntos. Os resultados estão expostos na Tabela 2.

Para verificar qual eletrodo descrimina mais, foi implementado um classificador para cada eletrodo. Analisando a Tabela 2, é possível observar que o parâmetro da dimensão da rede que gera os melhores resultados é $[1,100]$, ou seja, com 100 neurônios. Os eletrodos E2 e E6 tiveram

Tabela 2. Resultados da precisão (\%).

\begin{tabular}{l|l|l|l|l|l|l}
\hline Dim & E1 & E2 & E3 & E4 & E5 & E6 \\
\hline \hline$[1,3]$ & $58,2 \pm 1,4$ & $36,2 \pm 3,8$ & $38,7 \pm 1,7$ & $45,3 \pm 2,4$ & $35,9 \pm 3,0$ & $33,4 \pm 2,3$ \\
{$[1,10]$} & $74,5 \pm 1,6$ & $45,8 \pm 2,4$ & $55,7 \pm 2,6$ & $55,6 \pm 2,5$ & $49,8 \pm 3,2$ & $43,6 \pm 1,7$ \\
{$[1,25]$} & $88,0 \pm 1,6$ & $60,7 \pm 2,2$ & $70,8 \pm 2,1$ & $65,0 \pm 2,4$ & $70,6 \pm 2,3$ & $65,2 \pm 1,9$ \\
{$[1,50]$} & $91,8 \pm 0,8$ & $66,4 \pm 1,6$ & $80,2 \pm 1,5$ & $73,8 \pm 1,6$ & $78,2 \pm 2,0$ & $71,9 \pm 1,9$ \\
{$[1,100]$} & $95,4 \pm 1,1$ & $75,6 \pm 1,8$ & $85,5 \pm 1,1$ & $83,4 \pm 1,6$ & $86,2 \pm 1,0$ & $79,2 \pm 2,1$ \\
\hline
\end{tabular}

os piores percentuais de acurácia. Os eletrodo E3, E4 e E5 tiveram bons resultados, ficando entre 83,0 e $87,0 \%$ de classificações corretas. Já o eletrodo E1 teve o melhor percentual de classificação, com mais de 95,0\% de precisão. Vale ressaltar, que o desvio padrão desses resultados ficaram abaixo de $2,1 \%$, o que mostra pouca variância na classificação do modelo projetado.

A Tabela 3 traz a matriz de confusão do eletrodo E1 com dimensão $[1,100]$. Os percentuais ficaram a cima de $93,0 \%$ de precisão, o que mostra um percentual baixo de confusão. O maior percentual de confusão foi entre as amostras de GM e GD/ID, com 4,9\% de confusão entre si. Os demais ficaram abaixo de $2,7 \%$.

Tabela 3. Matriz de confusão do eletrodo E2 com dimensão [1, 100] (\%).

\begin{tabular}{l|lll}
\hline & GM & GD/ID & ID \\
\hline \hline GM & $93,3 \pm 2,3$ & $2,7 \pm 1,9$ & $0,4 \pm 0,6$ \\
GD $/$ ID & $4,9 \pm 2,1$ & $95,2 \pm 2,5$ & $2,0 \pm 1,1$ \\
ID & $1,8 \pm 1,1$ & $2,1 \pm 1,1$ & $97,6 \pm 1,1$ \\
\hline
\end{tabular}

Outro resultado que merece destaque, foi usando o eletrodo E1 mas com 50 neurônios $([1,50])$, atingindo média de 91,8\% de precisão. A Tabela 4 mostra a matriz de confusão dessa especificação. A substância GM teve 87,1\% de precisão e foi bastante confundida com GD/ID, com índices de 10,1\%. As amostras de GD/ID e ID tiveram boas classificações, ficando com percentuais acima de $91,0 \%$. Os demais valores de confusão entre as substâncias ficaram abaixo de $5,0 \%$.

Tabela 4. Matriz de confusão do eletrodo E2 com dimensão $[1,50](\%)$.

\begin{tabular}{l|lll}
\hline & GM & GD/ID & ID \\
\hline \hline GM & $87,1 \pm 2,3$ & $4,8 \pm 1,1$ & $0,3 \pm 0,5$ \\
GD/ID & $10,1 \pm 2,5$ & $91,1 \pm 2,0$ & $2,6 \pm 1,1$ \\
ID & $2,8 \pm 1,1$ & $4,0 \pm 1,7$ & $97,1 \pm 1,2$ \\
\hline
\end{tabular}

\section{CONCLUSÕES}

O sistema de LE é um instrumento analítico capaz de caracterizar amostras aquosas com composição complexa, combinando conhecimentos de vários ramos da ciência. Para isso, neste estudo usou-se técnicas de reconhecimento de padrões, como a rede SOM.

O objetivo geral do estudo foi investigar os resultados de impedância gerados por um sistema de LE usando redes SOM, com finalidade de identificar diferentes amostras de realçadores de sabor.

Com os métodos determinados e após a aplicação dos mesmos no banco de dados, os melhores resultados alcançados através de cálculos de precisão foram de 95,4\%. Os resultados obtidos indicam que a arquitetura do eletrodo E1 é o que propicia a maior descriminância dessas substâncias. 
Como trabalhos futuros, os autores têm como objetivo fazer novos testes envolvendo diferentes ferramentas de análise de dados e reconhecimento de padrões com o intuito de identificar os métodos que atingem o melhor resultado.

\section{AGRADECIMENTOS}

Agradecimento à CNPq (Conselho Nacional de Desenvolvimento Científico e Tecnológico) pela bolsa de mestrado concedida e a FAPEMIG (Fundação de Amparo à Pesquisa do Estado de Minas Gerais).

\section{REFERÊNCIAS}

Asan, U. and Ercan, S. (2012). Computational Intelligence Systems in Industrial Engineering: With Recent Theory and Applications, volume 6. Atlantis Press.

Beale, M.H., Hagan, M.T., and Demuth, H.B. (2012). Neural network toolbox 7 user's guide. In R2012a, The MathWorks Inc.

Breijo, E.G., Pinatti, C.O., Peris, R.M., Fillol, M.A., Martínez-Máñez, R., and Camino, J.S. (2013). Tnt detection using a voltammetric electronic tongue based on neural networks. Sensors and Actuators A: Physical, 192(Supplement C), $1-8$.

Cawley, G.C. and Talbot, N.L. (2003). Efficient leaveone-out cross-validation of kernel fisher discriminant classifiers. Pattern Recognition, 36(11), 2585 - 2592.

Cetó, X., González-Calabuig, A., Capdevila, J., PuigPujol, A., and del Valle, M. (2015). Instrumental measurement of wine sensory descriptors using a voltammetric electronic tongue. Sensors and Actuators B: Chemical, 207(Part B), 1053 - 1059.

Deisingh, A.K. and abnd Michael Thompson, D.C.S. (2004). Applications of electronic noses and tongues in food analysis. International Journal of Food Science and Technology, 39, $587-604$.

Duda, R.O., Hart, P.E., and Stork, D.G. (2001). Pattern Classification. Wiley-Interscience, 2 edition.

Fukushima, K.L. (2017). Desenvolvimento de sensores nanoestruturados para análise de realçadores de sabor em água. Master's thesis, Programa de Pós-Graduação em Engenharia de Biomateriais, Universidade Federal de Lavras.

Ghosh, H., Das, R., and RoyChaudhuri, C. (2016). Impedance based nanocrystalline silicon oxide immunosensor electronic tongue for ultrasensitive and low cost multiple food toxin detection. In IEEE International Instrumentation and Measurement Technology Conference Proceedings. IEEE.

Gutiérrez-Capitán, M., Vila-Planas, J., Llobera, A., Jiménez-Jorquera, C., Capdevila, F., Domingo, C., and Puig-Pujol, A. (2014). Hybrid electronic tongues based on microsensors applied to wine quality control. In SENSORS, 2014 IEEE, 2130-2133.

Kang, B.S., Lee, J., and Park, H.J. (2014). Electronic tongue-based discrimination of korean rice wines (makgeolli) including prediction of sensory evaluation and instrumental measurements. Food chemistry, 151, 31723.

Liu, M., Wang, M., Wang, J., and Li, D. (2013). Comparison of random forest, support vector machine and back propagation neural network for electronic tongue data classification: Application to the recognition of orange beverage and chinese vinegar. Sensors and Actuators B: Chemical, 177(Supplement C), $970-980$.

Lu, L., Hu, X., Tian, S., Deng, S., and Zhu, Z. (2016). Visualized attribute analysis approach for characterization and quantification of rice taste flavor using electronic tongue. In Analytica Chimica Acta, volume 919, 11 19. Elsevier.

Men, H., Ge, Z., Guo, Y., An, L., and Peng, Y. (2009). Biomimetic electronic tongue for classification of mineral water. Measuring Technology and Mechatronics Automation, International Conference on, 2, 621-624.

Milani, T.M.G. (2013). Estudo da aromatização préextrusão e das condições de extrusão sobre as características físicas e retenção de aroma em proteina isolada de soja. Master's thesis, Instituto de Biociências, Letras e Ciências Exatas, Universidade Estadual Paulista Julio de Mesquita Filho.

Nery, E.W. and Kubota, L.T. (2016). Integrated, paperbased potentiometric electronic tongue for the analysis of beer and wine. In Analytica Chimica Acta, volume 918, 60 - 68. Elsevier.

Peris, M. and Escuder-Gilabert, L. (2013). On-line monitoring of food fermentation processes using electronic noses and electronic tongues: a review. In Analytica chimica acta, volume 804, 29 - 36. Elsevier.

Saha, P., Ghorai, S., Tudu, B., Bandyopadhyay, R., and Bhattacharyya, N. (2016). Tea quality prediction by autoregressive modeling of electronic tongue signals. IEEE Sensors Journal, 16(11), 4470-4477.

Saha, P., Ghorai, S., Tudu, B., Bandyopadhyay, R., and Bhattacharyya, N. (2014). A novel technique of black tea quality prediction using electronic tongue signals. In IEEE Transactions on Instrumentation and Measurement, volume $63,2472-2479$. IEEE.

Sharma, P. and Ugale, S. (2015). Electronic tongue based liquid identification using lvq algorithm. In 2015 International Conference on Computer, Communication and Control (IC4), 1-6.

Silva, T.G. and Paixão, T.R.L.C. (2017). Development of an electronic tongue to distinguish cutting agents in cocaine samples to understand drug trafficking. In 2017 ISOCS/IEEE International Symposium on Olfaction and Electronic Nose (ISOEN), 1-3.

Vesanto, J., Himberg, J., Alhoniemi, E., and Parhankangas, J. (2000). Som toolbox for matlab 5. Helsinki University of Technology.

Wei, Z. and Wang, J. (2014). Tracing floral and geographical origins of honeys by potentiometric and voltammetric electronic tongue. In Computers and Electronics in Agriculture, volume 108, 112 - 122. Elsevier.

Wei, Z., Wang, J., and Zhang, X. (2013). Monitoring of quality and storage time of unsealed pasteurized milk by voltammetric electronic tongue. In Electrochimica Acta, volume 88, 231 - 239. Elsevier.

Zielinski, A.A.F., Haminiuk, C.W.I., Nunes, C.A., Schnitzler, E., Ruth, S.M., and Granato, D. (2014). Chemical composition, sensory properties, provenance, and bioactivity of fruit juices as assessed by chemometrics: a critical review and guideline. Comprehensive reviews in food science and food safety, 13(3), $300-316$. 